\title{
Visualización multi-escala de aciertos y errores de un mapa de usos de suelo: El caso de la cuenca del lago de Cuitzeo, Michoacán, México. ${ }^{\hat{\hbar}}$
}

\section{A multi-scale visualization of accuracies and inaccuracies of a land use map: the case of the Cuitzeo lake basin, Michoacan, Mexico.}

\author{
Stephane Couturier ${ }^{\mathrm{a},}$, Valdemar Coria Tapia ${ }^{\mathrm{a}}$, Yannick Deniau ${ }^{\mathrm{b}}$, Javier Osorno Covarrubias $^{\mathrm{a}}$ \\ ${ }^{a}$ Laboratorio de Análisis Geo-Espacial (LAGE), Instituto de Geografía, Universidad Nacional Autónoma de México, Circuito Exterior, Ciudad Universitaria, \\ Coyoacán, 04510, México, D. F. \\ ${ }^{b}$ Instituto Nacional de Estadística y Geografía (INEGI), Av. Héroe de Nacozari Sur, Núm. 2301, Fracc. Jardines del Parque, C.P. Aguascalientes, Ags. México.
}

\section{Resumen}

En la última década, un avance metodológico significativo ha permitido medir la fiabilidad de mapas regionales de usos de suelo en los paisajes altamente dinámicos de los países sub-tropicales. Lo anterior ha sido posible con un desarrollo geoestadístico y con la teoría de la lógica difusa, y ha involucrado la construcción de un acervo detallado de materiales cartográficos evaluados y de verificación. Sin embargo, la visualización de ambos materiales, de su comparación, y de la distribución espacial explícita de aciertos y errores derivados de estos estudios (con su enorme potencial visual-explicativo para la toma de decisiones con base en esta cartografía), no ha sido publicada. Presentamos aquí la visualización de aciertos y errores, con criterios de lógica difusa, del mapa de usos de suelo de alta taxonomía de la cuenca del lago de Cuitzeo, extraído de la cartografía a escala 1:250,000 del Inventario Forestal Nacional del año 2000 en México.

Palabras clave: Ordenamiento territorial; cobertura vegetal; abandono de cultivos; paisaje dinámico; lógica difusa; confiabilidad.

\begin{abstract}
In the last decade, a methodological breakthrough has permitted the accuracy assessment of regional land use maps of highly dynamic landscapes for sub-tropical countries. This has been achieved on the basis of geostatistics and fuzzy logics, and has led to a detailed database of spatial features derived from the assessed cartography and from the verification material. However, the visualization of these features, of their comparison, and of the spatial distribution of matches and mismatches (with an enormous explanatory potential for decision making based on this cartography), had not been published yet. We present the visualization of matches and mismatches at verification points, with fuzzy logic criteria, of the high taxonomy land use map of the Cuitzeo lake watershed, extracted from the 1:250:000 scale National Forest Inventory of year 2000 in Mexico.
\end{abstract}

Keywords: Land use planning; vegetation cover; field abandonment; dynamic landscape; fuzzy logic; accuracy assessment.

\footnotetext{
(c) Couturier Stephane, Valdemar Coria Tapia, Yannick Deniau \& Francisco Javier Osorno Covarrubias. Published by Terra Digitalis.

This is an Open Access article distributed under the terms of the Creative Commons Attribution License (https://creativecommons.org/licenses/by-nc-sa/4.0/), which permits non-commencial sharing of the work and adaptions, provided the original work is properly cited and the new creations are licensed under identical terms.
}

*E-mail address: andres@igg.unam.mx

\section{Introducción}

Una de las vocaciones de la cartografía de cobertura vegetal y usos de suelo (CVUS) a nivel regional es ser útil a un órgano de toma de decisiones para la planeación o el ordenamiento territorial / ecológico. Un atributo importante de esta cartografía es su fiabilidad, es decir qué tanto corresponden las categorías representadas en ella con la realidad, o bien qué cantidad de aciertos y de errores tienen estas categorías. En México, un avance metodológico significativo ha sido realizado en la última década en cuanto a medir la fiabilidad de mapas regionales de 
alta taxonomía (Couturier, 2007, 2012), utilizando los últimos avances en muestreos complejos, geoestadística y lógica difusa. En particular, el plan de desarrollo para la producción cartográfica del Inventario Forestal Nacional del año 2000 (IFN2000), elaborado en el Instituto de Geografía de la UNAM, ha involucrado la construcción de un acervo detallado de materiales cartográficos evaluados y de materiales de verificación (Mas et al., 2002). Con las recomendaciones por organismos internacionales en el sentido de adoptar estos métodos, el Instituto Nacional de Estadísticas y Geografía (INEGI) y la Comisión Nacional Forestal (CONAFOR) están considerando incorporar este tipo de material para producir cartografía estadísticamente robusta en México, aunque todavía no lo hacen (Martínez González y Couturier, 2016).

Partiendo de la propiedad sinóptica de la cartografía, argumentamos que la visualización espacial de aciertos y errores encima del mapa de CVUS, permite una comprensión más inmediata y una utilización de la información de fiabilidad por parte de usuarios del mapa como organizaciones sociales y/o sujetos de distintos niveles político-administrativos.

La cuenca cerrada del lago de Cuitzeo está ubicada al noreste del estado de Michoacán, México. En sus 400000 ha de superficie se encuentran usos de suelo como la agricultura de temporal anual, la agricultura de riego y el pastizal inducido. La cobertura vegetal original era principalmente bosque de encino, pino-encino y pino hacia el suroeste, y matorral subtropical hacia el noreste. La alta diversidad taxonómica a nivel sub-comunidad (21 clases) del mapa de CVUS del IFN2000, traduce paisajes muy fragmentados y dinámicos así como transiciones entre zonas climáticas, típicos de muchos escenarios sub-tropicales en México. Este mapa se ha evaluado con un enfoque difuso por Couturier et al. (2008), quienes presentan los resultados en la forma de matrices de error e índices de fiabilidad, pero el material de verificación con interpretación difusa generado para la evaluación, no se ha publicado como tal. Con el objetivo de extender la utilidad de los métodos de evaluación de fiabilidad, presentamos una combinación del mapa de interés con su material de verificación.

\section{Método}

La construcción de las capas representadas en el mapa deriva de un material de verificación descrito en una primera subsección (abajo), y responde a una secuencia de tres etapas, típica de un proceso de evaluación de fiabilidad de mapas de alta taxonomía (ver Couturier, 2012):

1. Diseño de muestreo en dos etapas (selección de marcos fotográficos y selección de puntos de verificación)

2. Interpretación de fotografías aéreas con lógica difusa

3. Comparación entre material de verificación e información del mapa.

\subsection{Material de verificación}

El material de verificación utilizado fue una cobertura completa de 244 fotografías aéreas en papel a escala 1:37,000, adquiridas en 1999, siendo éstas casi síncronas con las imágenes Landsat del año 2000, que sirvieron para la elaboración del IFN2000. El espacio-mapa representado fue elaborado con base en un compuesto a color de un mosaico de estas imágenes Landsat (bandas 5, 4 y 1) del año 2000, georreferenciadas a la cartografía topográfica del INEGI a 1:50,000.

Cuadros de aproximadamente 3 por $6 \mathrm{~km}$ se encuentran sobrepuestos a este mosaico y representan el área útil de fotografía de una cobertura aérea a 1:37,000, adquirida en el año 1999, georreferenciados también a la cartografía topográfica del INEGI a 1:50,000. Estas áreas útiles de fotografía sirvieron de material principal de verificación. La cartografía en los cuadros fue resultado de la interpretación difusa (ver subsección abajo) de la fotografía aérea y fue la base de la primera evaluación estadísticamente robusta en un país subtropical (Couturier et al., 2007).

\subsection{Diseño de muestreo en dos etapas}

Para evaluar la fiabilidad del mapa del IFN 2000 en la cuenca del lago de Cuitzeo, se aplicó un método de evaluación de fiabilidad en dos etapas (Couturier et al., 2008), para 21 clases del nivel subcomunidad, cartografiadas en la cuenca. El diseño de muestreo tiene como objetivo la selección de sitios estadísticamente representativos de cada clase del mapa. Para concentrar la muestra espacialmente y realizar así ahorros de recursos, se emplea un muestreo en dos etapas: Primero, se realiza una selección de Unidades Primarias de Muestreo (UPM), que corresponden a un área útil de fotografía aérea. Segundo, se realiza una selección de Unidades Secundarias de Muestreo (USM), que corresponden a puntos de verificación dentro de las UPM previamente seleccionadas. En el caso de las clases comunes (clases cuya superficie rebasa el $5 \%$ del mapa), se realizó una selección aleatoria simple en la primera etapa y una selección aleatoria estratificada en la segunda etapa. En el caso de las clases escasas (clases cuya superficie es inferior a $5 \%$ del mapa), se realizó una selección aleatoria proporcional a la frecuencia de la clase en cada foto (ver Couturier et al., 2008) en la primera etapa, y una selección aleatoria inversamente proporcional a esta frecuencia en la segunda etapa. Como elementos cartográficos resultado de este diseño, están los cuadros fotográficos seleccionados y los puntos de verificación.

\subsection{Interpretación de fotografías aéreas con lógica difusa}

La cuenca del lago de Cuitzeo comprende situaciones de complejidad del paisaje, típicas de zonas subtropicales como son: transición entre clima tropical seco y clima templado subhúmedo, áreas con actividad agrícola de roza, tumba y quema, transición espacial y temporal entre diferentes tipos de vegetación, paisajes muy fragmentados, etc. El desafío que estas situaciones representan, es la correcta caracterización de los puntos de verificación en el método de evaluación, pues no siempre 
es posible ni conveniente limitarse a una clase única para caracterizar el punto de verificación, porque este ejercicio puede ser subjetivo (Stehman y Czaplewski, 1998). Tales evaluaciones subjetivas del mapa llevan generalmente a subestimar su fiabilidad (Laba et al., 2002). Una estrategia empleada para tomar en cuenta la posible ambigüedad temática del sitio de verificación es el enfoque difuso lingüístico (Gopal y Woodcock, 1994), muy bien adaptado para la clasificación visual de fotografías. Las calificaciones difusas para etiquetar polígonos son las siguientes:

5. Esta clase corresponde perfectamente al polígono.

4. Esta clase corresponde bien al polígono.

3. Esta clase no es la mejor opción, pero se encuentra parcial o probablemente en el polígono.

2. Esta clase es una mala clasificación del polígono.

1. Esta clase es una clasificación totalmente inaceptable del polígono.

Así, el material de verificación fue basado en la foto-interpretación difusa de estéreo-pares analógicos. Primero, una delineación de polígonos homogéneos se realizó a escala 1:125 000 (escala más grande que la escala del IFN2000 y adecuada para foto-interpretación), en micas de interpretación. Segundo, esta interpretación se restituyó en pantalla sobre fondo del espaciomapa, permitiendo reducir errores geométricos del material de verificación respecto del mapa IFN2000. Tercero, cada punto seleccionado (USM) para verificación, se ubicó primero en el compuesto a color del mosaico Landsat, y luego se transfirió visualmente en la mica de foto-interpretación.

A cada polígono se le atribuyó una calificación lingüística (ver arriba), para las cuatro clases más probablemente representadas en el polígono. Todo este proceso se realizó sin el conocimiento del contenido temático del IFN2000 en los materiales utilizados. De este paso se deriva el conjunto de mini-mapas ("maplets") de polígonos, enmarcadas en las áreas útiles de las fotografías seleccionadas en el paso anterior, y cada polígono está representado con la etiqueta de la clase más probable contenida en él.

\subsection{Comparación entre material de verificación e informa- ción del mapa}

En cada punto de verificación, vía filtros de un Sistema de Información Geográfica (SIG), se realizó la comparación entre las etiquetas difusas de verificación y las etiquetas del mapa IFN2000. Para tomar en cuenta la escala de mapa, se aplicó una tolerancia de 500m entre la posición del USM y la posición de los polígonos cartografiados (tolerancia de posición). Para tomar en cuenta el carácter difuso del material de verificación, se utilizaron dos niveles de tolerancia temática para evaluar el mapa IFN2000: uno que tolera el conjunto de las clases con calificación superior o igual a 3, y otro que sólo tolera el conjunto de clases con calificación de 5. De este paso, se deriva la información sobre el grado de acierto o error, según el nivel de tolerancia, en cada punto de verificación del mapa.

\subsection{Visualización multi-escala de la fiabilidad}

Derivado de los pasos descritos arriba, se obtiene una representación de elementos cartográficos de verificación, basados en la interpretación visual de fotografías aéreas (ver Couturier et al., 2009), que comprenden los mini-mapas (nivel Unidades Primarias de Muestreo, o UPM), y los puntos de evaluación (nivel Unidades Secundarias de Muestreo, o USM). Proponemos esta representación, para una visualización multi-escala de aciertos y errores de un mapa, porque el usuario puede apreciar visualmente la fiabilidad del producto a una escala gruesa (comparando el mapa del IFN2000 con un mini-mapa) y, simultáneamente, a una escala fina (el mapa del IFN 2000, o un mini-mapa, y el color de los puntos de verificación).

Esta caracterización multi-escala de la fiabilidad de una cartografía, puede ser relacionada con la caracterización multiescala de un fenómeno ambiental (ver Galicia et al., 2014), en el sentido de que la información no es exhaustiva a escalas más locales (grandes áreas no tienen información), sin embargo, por el tipo de muestra que se realiza, esta información es un acercamiento suficientemente representativo del fenómeno y, en el caso de este estudio, un acercamiento estadísticamente robusto. $\mathrm{El}$ acervo completo de datos incluye el mapa IFN2000 a evaluar, un espacio-mapa (base geométrica de la producción del mapa y de los materiales de verificación), un conjunto de minimapas de verificación, y los sitios puntuales de verificación. La distribución de aciertos y errores se visualiza en los sitios puntuales, de acuerdo con tres niveles de tolerancia: un nivel bajo de tolerancia temática y posicional del material de verificación (para discriminar aciertos estrictos del mapa), un nivel bajo de tolerancia temática y alto de tolerancia posicional (aciertos con tolerancia posicional), y un nivel alto de tolerancia temática y posicional (aciertos con tolerancia posicional y temática, para discriminar errores estrictos del mapa).

\section{Análisis de Mapa}

El mapa estático que se observa (Lámina 1) es la superposición de tres elementos cartográficos: el espacio-mapa, el conjunto de mini-mapas ("maplets") con los polígonos de verificación (etiquetados de la clase más probable contenida en él), y los puntos de verificación etiquetados del resultado de la evaluación difusa. Varios aspectos relacionados con la teoría de la evaluación de fiabilidad y los resultados de este estudio, se pueden apreciar con la simple visualización del mapa.

En primera instancia, esta superposición particular, propone una lectura multi-escala de la fiabilidad para el productor del mapa, dado que los errores del mapa se relacionarán visualmente con el material de verificación (los mini-mapas de verificación). Otra superposición (el mapa IFN2000 con los puntos de verificación), permitirá una lectura multi-escala de la fiabilidad para el usuario del mapa. 
VISUALIZACIÓN MULTI-ESCALA DE ACIERTOS Y ERRORES DE UN MAPA DE USOS DE SUELO: EL CASO DE LA CUENCA DEL LAGO DE CUITZEO, MICHOACÁN, MÉXICO. Autores: Stephane Couturier (1). Valdemar Coria Tapia (2). Yannick Deniau (1). Javier Osorno Covarrubias (1)

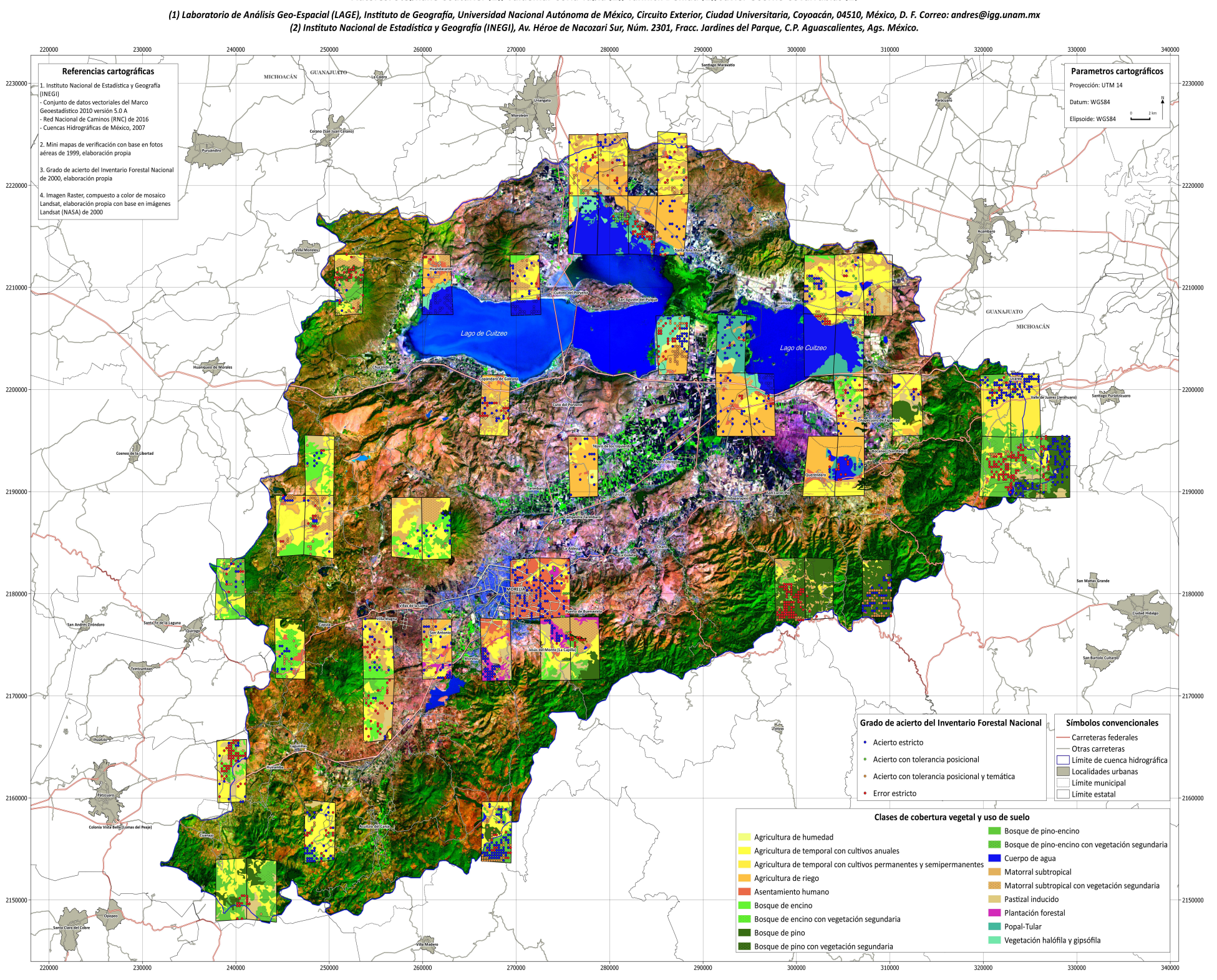

Lámina 1: Visualización multi-escala de aciertos y errores de un mapa de usos de suelo: El caso de la cuenca del lago de Cuitzeo, Michoacán, México Plate1. Multi-scale visualization of correct and erroneous verification points of the landuse map of the Cuitzeo lake watershed in Michoacan, Mexico. 
En segunda instancia, la densidad de aciertos en una determinada clase (color) de los mini-mapas, se relaciona con el índice de fiabilidad por clase de esta categoría (una alta densidad de puntos azules indicará una alta fiabilidad estricta de la clase observada). Por ejemplo, las clases de agricultura de riego, plantación forestal y asentamiento humano son de alta fiabilidad, mientras la clase de agricultura de temporal está asociada con baja fiabilidad.

En tercera instancia, la densidad espacial de los puntos de verificación para una determinada clase, indica la calidad de la representatividad espacial de la muestra realizada para esta clase; una aglutinación fuerte de puntos indica una representatividad limitada (alta autocorrelación espacial), o bien refleja una distribución geográfica limitada de la clase muestreada. En nuestro estudio, 14 de las 21 clases mapeadas se encuentran con representación escasa en la cuenca, y Couturier et al. (2008) comentan que por esta razón, la distribución espacial de los puntos de verificación es muy densa. A este respecto, el diseño de evaluación está previsto para una aplicación a nivel nacional para la cual no habría este problema. En contraste, la clase de uso de suelo de agricultura de riego, y la clase de bosque de encino, parecen estar muestreadas con buena representatividad espacial. En cuarta instancia, finalmente, el color de los puntos de verificación sugiere una dificultad o no de la identificación de la clase en el material de verificación (foto aérea).

\section{Conclusión}

Este artículo propone un modelo cartográfico dirigido a documentar visualmente la información de fiabilidad que acompaña los mapas de usos de suelo. Esta cartografía consiste en la combinación interactiva del mapa de interés, con un material de verificación basado en un muestreo doble y una interpretación con lógica difusa. Aplicamos este modelo cartográfico al mapa de usos de suelo de alta taxonomía de la cuenca del lago de Cuitzeo, extraído de la cartografía a escala 1:250,000 del Inventario Forestal Nacional del año 2000 (IFN2000) en México. Los índices de fiabilidad y los patrones de error en clases del IFN2000, fueron registrados en forma de una matriz de confusión en Couturier et al. (2008). Aquí, el modelo permite una variedad de superposición para una apreciación visual de la fiabilidad. Explicamos el potencial explicativo del modelo en su modo "fiabilidad para el productor", utilizando la superposición de los mini-mapas de verificación y de los puntos de verificación. Este modo permite resaltar el alto nivel de fiabilidad de las clases "agricultura de riego", "plantación forestal", y "asentamiento humano", pero también una alta densidad espacial de los aciertos y errores para una mayoría de clases, lo que alerta al usuario acerca de que los valores de fiabilidad pueden contener una alta incertidumbre debido a la auto-correlación espacial. Una clase con alta densidad de puntos café para la clase "matorral sub-tropical" indica posiblemente una dificultad de interpretación de esta cobertura en las fotografías aéreas (se necesita mayor tolerancia temática para encontrar una coincidencia entre el mapa y el material de verificación).
Estas apreciaciones, además de las matrices cuantitativas de confusión, ayudan a quienes elaboran los mapas, a buscar una mejor estrategia de producción cartográfica o de verificación cartográfica (otro sensor, otra resolución, u otro sistema clasificatorio).

La información contenida en el modelo también es útil para el tomador de decisiones, quien elige confiar en la representación del mapa, o bien recurrir a un esfuerzo de producción cartográfica alternativo. Por ejemplo, para establecer una cartografía base del proceso de abandono de la agricultura de temporal en Michoacán, una Organización No Gubernamental, revisando estos datos, optaría por buscar fuentes alternativas de mapeo a la cartografía del IFN2000. Este trabajo contribuye a sostener que las nuevas tecnologías de la información geográfica, abren posibilidades interesantes en términos de apropiación del concepto de fiabilidad cartográfica, por parte de una gama cada vez más extensa de usuarios.

\section{Datos}

La información de mayor valor agregado derivada de la investigación, la constituyen datos espaciales en formato vectorial (polígonos y puntos). La información está contenida en un sistema de información geográfica (QGIS, versión 2.14.3) con las características contenidas en la Tabla 1:

Tabla 1: Datos espaciales Table 1: Spatial data

\begin{tabular}{lc}
\hline & Información de los datos espaciales \\
\hline Estructura del dato & Vector \\
Tipo del dato & Puntos y polígonos \\
Número total de puntos & 2,023 \\
Número total de polígonos & 727 \\
\hline \multicolumn{1}{c}{ Parámetros cartográficos principales } \\
\hline Proyección cartográfica & Universal Transversa de Mer- \\
& cator (UTM), zona 14N \\
Datum: & WGS 84 \\
\hline
\end{tabular}

\section{Software}

La capa raster fue generada con el software de Licencia ENVI, versión 6.0. Las capas vectoriales fueron generadas con dos SIGs: Arc View, versión 3.2, y ArcGIS versión 9.3. El diseño del mapa fue realizado con QGIS, versión 2.14.3.

\section{Agradecimientos}

Los autores agradecen a Erna López Granados por haber proporcionado la cobertura de fotografías aéreas de la cuenca del lago de Cuitzeo. La porción de investigación relativa al procesamiento de los datos espaciales, se realizó en el ámbito del proyecto CONACYT núm. 38965T, por lo cual se le agradece a los fondos públicos de investigación en México. La parte 
del diseño del mapa y de su análisis se realizó en el ámbito del proyecto PAPIIT IN302417 "Seguridad alimentaria versus preservación ambiental: diseño de una plataforma cartográfica nacional para analizar su compatibilidad de forma multiescalar". Se agradece a varios ejidatarios de la tercera edad, del pueblo El Cerro, en las inmediaciones del lago de Cuitzeo, por proveer información sobre dinámicas comunes del paisaje en la zona tropical seca de la cuenca.

\section{Diseño del mapa}

Un aspecto importante de la edición cartográfica consistió en harmonizar los colores y contrastes entre el compuesto a color de las imágenes Landsat, la leyenda de los mapas de verificación (Unidades Primarias de Muestreo), y el color de los sitios de verificación (Unidades Secundarias de Muestreo).

\section{Referencias}

Couturier, S., 2007. Evaluación de errores de cartas de cobertura vegetal y usos del suelo con enfoque difuso y con la simulación de imágenes de satélite. Tesis de Doctorado, UNAM/Université Paul DSabatier de Tolouse, Francia (UPS), México.

Couturier, S., 2012. The quality of detailed land cover maps in highly biodiverse areas: lessons learned from the Mexican experience. En: Diez, J.J. (Eds.), Sustainable Forest Management - Current Research, Intech Open Access Publishing, ISBN 978-953-51-0621-0.

Couturier, S., Mas, J.-F., Cuevas, G., Benítez, J., Vega-Guzmán, A., CoriaTapia, V., 2009. An accuracy index with positional and thematic fuzzy bounds for land-use/ land-cover maps. Photogrammetric Engineering and Remote Sensing 75 (7), 789-805.

Couturier, S., Mas, J.-F., Vega, A., Tapia, V., 2007. Accuracy assessment of land cover maps in sub-tropical countries: a sampling design for the Mexican National Forest Inventory map. Online Journal of Earth Sciences 1 (3), 127135, ISSN: 1991-7708.
Couturier, S., Vega, A., Mas, J. F., Tapia, V., López-Granados, E., 2008. Evaluación de confiabilidad del mapa del Inventario Forestal Nacional 2000: diseños de muestreo y caracterización difusa de paisajes. Investigaciones Geográficas (UNAM), 67, 20-38.

Galicia, L., Cuevas Fernández, M. L., González Ramírez, L. M., Couturier, S., 2014. Detection of environmental change in tropical and temperate forests of Mexico with Remote Sensing: a multi-scale time and space approach. Interciencia 39 (6), 368-374.

Gopal, S., Woodcock, C., 1994. Accuracy of thematic maps using fuzzy sets I: theory and methods. Photogrammetric Engineering and Remote Sensing (58), 35-46.

Laba, M., Gregory, S., Braden, J., Ogurcak, D., Hill, E., Fegraus, E., Fiore, J., DeGloria, S. D., 2002. Conventional and fuzzy accuracy assessment of the New York Gap Analysis Project land cover map. Remote Sensing of Environment (81), 443-455.

Martínez González, R., Couturier, S., 2016. Métodos geoestadísticos y enfoque difuso para el monitoreo forestal en México: hacia la medición robusta de la deforestación global. En: Moncada Maya J.O. y A. López López (coordinadores generales), Geografía de México. Una reflexión espacial contemporánea. Tomo I. Instituto de Geografía - UNAM, Ciudad de México, pp. 297-309, ISBN: 978-607-02-8276-8.

Mas, J. F., Velázquez, A., Palacio-Prieto, J. L., Bocco, G., Peralta, A., Prado, J., 2002. Assessing forest resources in Mexico: Wall-to-wall land use/ cover mapping. Photogrammetric Engineering and Remote Sensing 68 (10), 966969.

Stehman, S. V., Czaplewski, R. L., 1998. Design and analysis for thematic map accuracy assessment: fundamental principles. Remote Sensing of Environment (64), 331-344.

This article accompanies the following material:

HTML: $\quad$ DOI: 10.22201/igg.terradigitalis.2017.1.5.24

Static map: $\quad$ DOI: 10.22201/igg.terradigitalis.2017.1.5.52

3D map: $\quad$ DOI: 10.22201/igg.terradigitalis.2017.1.5.59

Interactive map: DOI: 10.22201/igg.terradigitalis.2017.1.5.64 